\title{
Finite and Infinite Arithmetic Progressions Related to Beta-Expansion
}

\author{
Bing $\mathrm{Li}^{1}$ and Chao $\mathrm{Ma}^{2}$ \\ ${ }^{1}$ Department of Mathematics, South China University of Technology, Guangzhou 510640, China \\ ${ }^{2}$ Department of General Education, Macau University of Science and Technology, Macau \\ Correspondence should be addressed to Chao Ma; fractalma@sina.com
}

Received 8 May 2014; Accepted 9 August 2014; Published 28 August 2014

Academic Editor: Douglas R. Anderson

Copyright (c) 2014 B. Li and C. Ma. This is an open access article distributed under the Creative Commons Attribution License, which permits unrestricted use, distribution, and reproduction in any medium, provided the original work is properly cited.

Let $1<\beta<2$ and $\varepsilon(x, \beta)$ be the $\beta$-expansion of $x \in[0,1)$. Denote by $A_{\beta}(x)$ the set of positions where the digit 1 appears in $\varepsilon(x, \beta)$. We consider the sets of points $x$ such that $A_{\beta}(x)$ contains arbitrarily long arithmetic progressions and includes infinite arithmetic progressions, respectively. Their sizes are investigated from the topological, metric, and dimensional viewpoints.

\section{Introduction}

Let $A$ be a sequence of natural numbers. The lower asymptotic density $\rho(A)$ of $A$ (the upper asymptotic density $\bar{\rho}(A)$ of $A$, resp.) is $\bar{d}$ efined as

$$
\begin{gathered}
\underline{\rho}(A)=\liminf _{N \rightarrow \infty} \frac{\sum_{n \leq N, n \in A} 1}{N}, \\
\left(\bar{\rho}(A)=\limsup _{N \rightarrow \infty} \frac{\sum_{n \leq N, n \in A} 1}{N}, \text { resp. }\right) .
\end{gathered}
$$

If $\rho(A)=\bar{\rho}(A)$, the common value is called the asymptotic density of $A$, denoted by $\rho(A)$.

The concept of asymptotic density was extensively applied to number theory such as arithmetic progressions, the first of which is the following theorem due to Szemerédi [1].

Theorem 1 (see [1]). If $\bar{\rho}(A)>0$, then the sequence A contains arbitrarily long arithmetic progressions.

The issue to find what kind of sequence $A$ can contain arbitrarily long arithmetic progressions is very popular nowadays. For instance, Green and Tao [2] proved that the sequence $P$ of prime numbers contains arbitrarily long arithmetic progressions, which extends Theorem 1 since $\rho(P)=0$.

Assume that the sequence $A$ contains arbitrarily long arithmetic progressions; a further question is whether $A$ can contain an infinite arithmetic progression. In 1972, Wagstaff Jr. [3] found a sequence of natural numbers which contains arbitrarily long arithmetic progressions but does not have any infinite one. In fact, he even gave more information as the following.

Theorem 2 (see [3]). For each $a, b \in[0,1]$ with $a \leq b$, there exists a sequence of natural numbers $A$ such that $A$ contains no infinite arithmetic progression and $\rho(A)=a$ and $\bar{\rho}(A)=b$.

The topics of arithmetic progressions and binary expansion were firstly linked by Šalát and Tomanová [4]. They considered the sequence of the positions where the digit 1 appears in the binary expansion of real numbers in the unit interval. In this paper, we investigate the same questions for $\beta$-expansion with $1<\beta \leq 2$ and generalize the results in [4].

Let $1<\beta \leq 2$. The $\beta$-transformation $T_{\beta}:[0,1) \rightarrow[0,1)$ is defined as $T_{\beta}(x)=\beta x(\bmod 1)$ for any $x \in[0,1)$. Consider the greedy $\beta$-expansion of $x$ :

$$
x=\frac{\varepsilon_{1}(x, \beta)}{\beta}+\frac{\varepsilon_{2}(x, \beta)}{\beta^{2}}+\cdots+\frac{\varepsilon_{n}(x, \beta)}{\beta^{n}}+\cdots,
$$

with $\varepsilon_{1}(x, \beta)=[\beta x]$ and $\varepsilon_{n}(x, \beta)=\varepsilon_{1}\left(T_{\beta}^{n-1}(x), \beta\right)$ for all $n \geq$ 2 , called $\beta$-digits of $x$. Here $[y]$ denotes the integer part of $y \in \mathbb{R}$. It is clear that all the digits $\varepsilon_{n}(x, \beta) \in\{0,1\}$ for any $x \in[0,1)$ and $n \geq 1$. 
Define

$$
A_{\beta}(x)=\left\{n \geq 1: \varepsilon_{n}(x, \beta)=1\right\}
$$

that is, the set of natural numbers with $\varepsilon_{n}(x, \beta)=1$. In other words, $A_{\beta}(x)$ is the sequence of positions where the digit 1 appears in the $\beta$-expansion of $x$.

Denote

$$
\begin{aligned}
& \mathscr{F}_{\beta}=\{ x \in[0,1): \text { the sequence } A_{\beta}(x) \text { contains } \\
&\text { arbitrarily long arithmetic progressions }\}, \\
& \mathscr{I}_{\beta}=\left\{x \in[0,1): \text { the sequence } A_{\beta}(x)\right. \text { contains } \\
&\text { infinite arithmetic progressions }\} .
\end{aligned}
$$

Obviously, $\mathscr{I}_{\beta} \subset \mathscr{F}_{\beta}$ since an infinite arithmetical progression contains arbitrarily long ones. In Section 2, we will give some introduction to $\beta$-expansion. In Sections 3 and 4 , we will describe the sets $\mathscr{F}_{\beta}, \mathscr{I}_{\beta}$ and their complements $\mathscr{F}_{\beta}^{c}$, $\mathscr{I}_{\beta}^{c}$ from the viewpoints of topology, metric, and Hausdorff dimension.

\section{Preliminary for $\beta$-Expansion}

When $\beta=2$, any sequence in $\{0,1\}^{\mathbb{N}}$ except countably many elements is a $\beta$-expansion of some point $x \in[0,1)$. It is not this case when $1<\beta<2$ is not an integer. Denote

$$
\begin{aligned}
& \Sigma_{\beta}=\left\{\omega \in\{0,1\}^{\mathbb{N}}: \omega\right. \text { is } \\
&\text { a } \beta \text {-expansion of some } x \in[0,1)\} .
\end{aligned}
$$

A $0-1$ sequence $\omega$ is said to be admissible if $\omega \in \Sigma_{\beta}$ and a 0 -1 word $\left(\varepsilon_{1}, \ldots, \varepsilon_{n}\right)$ is admissible if there exists one point $x \in[0,1)$ such that $\varepsilon_{1}(x, \beta)=\varepsilon_{1}, \ldots, \varepsilon_{n}(x, \beta)=\varepsilon_{n}$. Denote by $\Sigma_{\beta}^{n}$ the set of all admissible words of length $n$. Let $S_{\beta}$ be the closure of $\Sigma_{\beta}$ and then $\left(S_{\beta}, \sigma\right)$ is a subshift of $\left(\{0,1\}^{\mathbb{N}}, \sigma\right)$, where $\sigma$ is the shift transformation of the symbolic space $\{0,1\}^{\mathbb{N}}$. Parry [5] gave a criterion to check if a sequence in $\{0,1\}^{\mathbb{N}}$ is admissible where the $\beta$-expansion of the number 1 (defined similarly with (2)) plays an essential role. If the $\beta$ expansion of 1 , denoted by $\varepsilon(1, \beta)$, is finite, that is, ending with zeros, we call the modified periodic sequence $\varepsilon^{*}(1, \beta):=$ $\left(\varepsilon_{1}(1, \beta), \ldots, \varepsilon_{n-1}(1, \beta), \varepsilon_{n}(1, \beta)-1\right)^{\infty}$ the infinite $\beta$-expansion of 1 , where $\varepsilon_{n}(1, \beta) \neq 0$ is the last nonzero element. If $\varepsilon(1, \beta)$ is infinite, namely, that there are infinitely many $1 \mathrm{~s}$ in the sequence $\varepsilon(1, \beta)$, then just let $\varepsilon^{*}(1, \beta)=\varepsilon(1, \beta)$. We use the notation $\prec$ to represent the lexicographical order in the symbolic space $\{0,1\}^{\mathbb{N}}$.

Theorem 3 (see [5]). Let $\omega \in\{0,1\}^{\mathbb{N}}$. Then

$$
\omega \in \Sigma_{\beta} \text { iff } \sigma^{k} \omega \prec \varepsilon^{*}(1, \beta), \quad \forall k \geq 0 .
$$

For the case $\beta=2, S_{\beta}=\{0,1\}^{\mathbb{N}}$; that is, the coding space is the full shift space. When $1<\beta<2, S_{\beta}$ is a proper subset of $\{0,1\}^{\mathbb{N}}$. If $\varepsilon(1, \beta)$ is finite, $S_{\beta}$ is a subshift of finite type (SFT) which can be obtained by forbidding finitely many words. The set of $\beta$ 's with $S_{\beta}$ being SFT is dense in $(1,2]$ (see [5]). If $1<$ $\beta_{1}<\beta_{2}$, then $\Sigma_{\beta_{1}} \subset \Sigma_{\beta_{2}}$ and $S_{\beta_{1}} \subset S_{\beta_{2}}$, which means that every $\beta_{1}$-admissible sequence is $\beta_{2}$-admissible.

Definition 4. Let $\left(\varepsilon_{1}, \ldots, \varepsilon_{n}\right) \in \Sigma_{\beta}^{n}$. The set

$$
J\left(\varepsilon_{1}, \ldots, \varepsilon_{n}\right):=\left\{x \in[0,1): \varepsilon_{1}(x, \beta)=\varepsilon_{1}, \ldots, \varepsilon_{n}(x, \beta)=\varepsilon_{n}\right\}
$$

is called a cylinder of order $n$.

Any cylinder $J\left(\varepsilon_{1}, \ldots, \varepsilon_{n}\right)$ is a left-closed and right-open interval and its length

$$
\left|J\left(\varepsilon_{1}, \ldots, \varepsilon_{n}\right)\right| \leq \beta^{-n} .
$$

We say that a cylinder $J\left(\varepsilon_{1}, \ldots, \varepsilon_{n}\right)$ is full if $T_{\beta}^{n} J\left(\varepsilon_{1}, \ldots, \varepsilon_{n}\right)=$ $[0,1)$; that is, any admissible sequence can be concatenated with the word $\left(\varepsilon_{1}, \ldots, \varepsilon_{n}\right)$. In other words, $\left(\varepsilon_{1}, \ldots, \varepsilon_{n}, \omega\right) \in \Sigma_{\beta}$ if $\omega \in \Sigma_{\beta}$. We remark that $\left|J\left(\varepsilon_{1}, \ldots, \varepsilon_{n}\right)\right|=\beta^{-n}$ if $J\left(\varepsilon_{1}, \ldots, \varepsilon_{n}\right)$ is full. The following lemma, due to $\mathrm{Li}$ and $\mathrm{Wu}$ [6], will be used to prove Proposition 6.

Lemma 5 (see [6]). Let $\left(\varepsilon_{1}, \ldots, \varepsilon_{n}\right) \in \Sigma_{\beta}^{n}$. Then there exists $M>0$ such that the cylinder $J\left(\varepsilon_{1}, \ldots, \varepsilon_{n}, 0^{M}\right)$ is full, where $M$ just depends on $n$ and $\beta ; 0^{M}$ means the word of $M$ consecutive zeros.

When $\beta=2$, the Lebesgue measure $\mathscr{L}$ is a $T_{\beta}$-invariant measure. If $\beta$ is not an integer, the Lebesgue measure $\mathscr{L}$ is not $T_{\beta}$-invariant; however Rényi [7] proved that there exists a unique invariant measure $\mu_{\beta}$ equivalent to $\mathscr{L}$. Parry [5] and Gel'fond [8] independently gave the density formula of $\mu_{\beta}$ and $\mathscr{L}$

The function $\varepsilon(\cdot, \beta): x \mapsto \varepsilon(x, \beta)$ maps $x \in[0,1]$ to its $\beta$-expansion. Conversely there is a projection $\pi_{\beta}$ from the subshift $S_{\beta}$ to $[0,1]$ defined as $\pi_{\beta}(\omega)=\sum_{i=1}^{\infty}\left(\omega_{i} / \beta^{i}\right)$ for any $\omega=\left(\omega_{1}, \ldots, \omega_{i}, \ldots\right) \in S_{\beta}$. In particular, $\pi_{\beta} \varepsilon(x, \beta)=x$. We have the following commutative diagram; that is, $T_{\beta} \circ \pi_{\beta}=$ $\pi_{\beta} \circ \sigma$ :

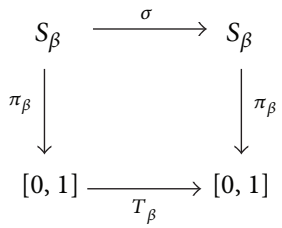

\section{Topological Properties of $\mathscr{F}_{\beta}$ and $\mathscr{I}_{\beta}$}

Let $N_{n}(x, \beta)=\sum_{k=1}^{n} \varepsilon_{k}(x, \beta)$ be the sum of first $n$ digits of the $\beta$-expansion of $x \in[0,1]$. Define

$$
E=\left\{x \in[0,1): \lim _{n \rightarrow \infty} \frac{N_{n}(x, \beta)}{n}=0\right\} .
$$


Proposition 6. The set $E$ is of the first category.

Proof. The set $E$ can be written as

$$
\begin{aligned}
E & =\bigcap_{k=1}^{\infty} \bigcup_{N=1}^{\infty} \bigcap_{n=N}^{\infty}\left\{x \in[0,1): \frac{N_{n}(x, \beta)}{n}<\frac{1}{k}\right\} \\
& =: \bigcap_{k=1}^{\infty} \bigcup_{N=1}^{\infty} \bigcap_{n=N}^{\infty} E_{k, n} .
\end{aligned}
$$

For any $k, n \in \mathbb{N}$, the set $E_{k, n}$ is the union of some cylinders $J\left(\varepsilon_{1}, \ldots, \varepsilon_{n}\right)$ of order $n$ satisfying $\varepsilon_{1}+\cdots+\varepsilon_{n}<n / k$. Note that the cylinder $J\left(\varepsilon_{1}, \ldots, \varepsilon_{n}\right)$ is a left-closed and right-open interval; denote by $\bar{J}\left(\varepsilon_{1}, \ldots, \varepsilon_{n}\right)$ its closure, which is obtained by adding the right endpoint of $J\left(\varepsilon_{1}, \ldots, \varepsilon_{n}\right)$. Let

$$
\bar{E}_{k, n}=\bigcup_{\varepsilon_{1}+\cdots+\varepsilon_{n}<n / k} \bar{J}\left(\varepsilon_{1}, \ldots, \varepsilon_{n}\right) .
$$

Choose $K \geq 1$ large enough such that there exists $x_{0} \in[0,1)$ with $\lim _{n \rightarrow \infty}\left(N_{n}\left(x_{0}, \beta\right) / n\right)>1 / K$. Such existence is because the sequence $u:=\left(10^{m}\right)^{\infty}$ is admissible where $m \geq 2$ with $\varepsilon_{m}^{*}(1, \beta)=\varepsilon_{1}^{*}(1, \beta)=1$ and $\lim _{n \rightarrow \infty}\left(N_{n}\left(\pi_{\beta}(u), \beta\right) / n\right)=$ $1 /(m+1)>0$. Notice that

$$
E \subset \bigcup_{N=1}^{\infty} \bigcap_{n=N}^{\infty} \bar{E}_{K, n} .
$$

Since the set $\bar{E}_{K, n}$ is closed, $\cap_{n=N}^{\infty} \bar{E}_{K, n}$ is a closed set; it suffices to show that the interior of $\cap_{n=N}^{\infty} \bar{E}_{K, n}$ is empty. Suppose it is not empty; then there exists one interval inside $\cap_{n=N}^{\infty} \bar{E}_{K, n}$, which implies that there exists some cylinder $J\left(\varepsilon_{1}, \ldots, \varepsilon_{m}\right)$ lying in this interval and then $J\left(\varepsilon_{1}, \ldots, \varepsilon_{m}\right) \subset \cap_{n=N}^{\infty} \bar{E}_{K, n}$. Thus there exists $M \in \mathbb{N}$ only depending on $m$ and $\beta$ such that the cylinder $J\left(\varepsilon_{1}, \ldots, \varepsilon_{m}, 0^{M}\right)$ is full by Lemma 5 . Therefore the sequence $\left(\varepsilon_{1}, \ldots, \varepsilon_{m}, 0^{M}, \varepsilon_{1}\left(x_{0}, \beta\right), \ldots, \varepsilon_{i}\left(x_{0}, \beta\right), \ldots\right)$ is admissible; put

$$
\begin{aligned}
y & =\pi_{\beta}\left(\varepsilon_{1}, \ldots, \varepsilon_{m}, 0^{M}, \varepsilon_{1}\left(x_{0}, \beta\right), \ldots, \varepsilon_{i}\left(x_{0}, \beta\right), \ldots\right) \\
& \in J\left(\varepsilon_{1}, \ldots, \varepsilon_{m}\right) .
\end{aligned}
$$

Thus $y \in \cap_{n=N}^{\infty} \bar{E}_{K, n}$ which implies $\lim \sup _{n \rightarrow \infty}\left(N_{n}(y, \beta) /\right.$ $n) \leq 1 / K$ contradicting

$$
\lim _{n \rightarrow \infty} \frac{N_{n}(y, \beta)}{n}=\lim _{n \rightarrow \infty} \frac{N_{n}\left(x_{0}, \beta\right)}{n}>\frac{1}{K} .
$$

So we complete the proof.

Remark 7. By the similar argument with the proof of Proposition 6, we can prove that the set

$$
E_{\alpha}=\left\{x \in[0,1): \lim _{n \rightarrow \infty} \frac{N_{n}(x, \beta)}{n}=\alpha\right\}
$$

is of the first category for any $0 \leq \alpha \leq 1$. When $1<\beta<2$, the set $E_{\alpha}$ may be empty. For instance, $\beta=(\sqrt{5}+1) / 2$; the set $\Sigma_{\beta}$ can be characterized as that

$\omega \in \Sigma_{\beta}$ iff the word 11 does not appear in $\omega$;

$$
\omega \text { does not end with } 101010 \ldots
$$

So $\lim \sup _{n \rightarrow \infty}\left(N_{n}(x, \beta) / n\right) \leq 1 / 2$ for any $x \in[0,1]$, which indicates $E_{\alpha}=\emptyset$ if $\alpha>1 / 2$.

Theorem 8. The set $\mathscr{F}_{\beta}$ is residual in $[0,1)$.

Proof. Firstly we claim that $\mathscr{F}_{\beta}^{c} \subset\left\{x \in[0,1): \rho\left(A_{\beta}(x)\right)=\right.$ $0\}$. Indeed, if $\rho\left(A_{\beta}(x)\right)>0$, by Theorem 1 , we know that the sequence $A_{\beta}(x)$ contains arbitrarily long arithmetical progressions; that is, $x \in \mathscr{F}_{\beta}$.

Secondly, combining the facts that

$$
\begin{aligned}
\mathscr{F}_{\beta}^{c} & \subset\left\{x \in[0,1): \rho\left(A_{\beta}(x)\right)=0\right\} \\
& =\left\{x \in[0,1): \lim _{n \rightarrow \infty} \frac{N_{n}(x, \beta)}{n}=0\right\}=E
\end{aligned}
$$

and the set $E$ is of the first category (Proposition 6), we know that the set $\mathscr{F}_{\beta}^{c}$ is of the first category. So the set $\mathscr{F}_{\beta}$ is residual.

Theorem 9. The set $\mathscr{I}_{\beta}$ is of the first category in $[0,1)$.

Proof. Let $A_{\beta}(x)=\left\{a_{1}(x)<a_{2}(x)<a_{3}(x)<\cdots<a_{n}(x)<\right.$ $\cdots\}$ and

$$
\begin{aligned}
& B=\{x \in[0,1): \text { the gap sequence } \\
&\left.\left\{a_{n+1}(x)-a_{n}(x)\right\}_{n \geq 1} \text { is bounded }\right\} .
\end{aligned}
$$

Then $\mathscr{I}_{\beta} \subseteq B$. Note that $B=\bigcup_{m=1}^{\infty} B_{m}$, where

$$
B_{m}=\left\{x \in[0,1):\left|a_{n+1}(x)-a_{n}(x)\right| \leq m \text {, for any } n \geq 1\right\} .
$$

It suffices to show that, for any $m \geq 1$, the set $B_{m}$ is nowhere dense. That is, for any interval $I \subset[0,1)$, there exists an interval $I^{\prime} \subset I$ such that $I^{\prime} \cap B_{m}=\emptyset$. Indeed, for given $I \subset[0,1)$, we can find a cylinder $J\left(\varepsilon_{1}, \varepsilon_{2}, \ldots, \varepsilon_{n}\right)$ such that $J\left(\varepsilon_{1}, \varepsilon_{2}, \ldots, \varepsilon_{n}\right) \subset I$ when $n$ is large enough. Put $I^{\prime}=J\left(\varepsilon_{1}, \varepsilon_{2}, \ldots, \varepsilon_{n}, 0^{m+1}\right)$. Obviously $I^{\prime} \subset I$. Since, for any $x \in I^{\prime}$, the word of $m+1$ consecutive zeros appears in the $\beta$ expansion of $x$, we have $I^{\prime} \cap B_{m}=\emptyset$. Therefore $B_{m}$ is nowhere dense. ing.

The combination of Theorems 8 and 9 implies the follow-

Corollary 10. The set $\mathscr{F}_{\beta} \backslash \mathscr{I}_{\beta}$ is residual in $[0,1)$.

\section{Metric and Dimensional Properties of $\mathscr{F}_{\beta}$ and $\mathscr{I}_{\beta}$}

Theorem 11. $\mathscr{L}\left(\mathscr{F}_{\beta}\right)=1$ and $\mathscr{L}\left(\mathscr{I}_{\beta}\right)=0$. 
Proof. Note that $\sum_{k=1}^{n} \varepsilon_{k}(x, \beta)=\sum_{k=0}^{n-1} \chi_{[1 / \beta, 1)}\left(T_{\beta}^{k} x\right)$, where $\chi$ is the indicator function. By Birkhoff's ergodic theorem, we have, for $\mu_{\beta}$-almost every (a.e.) $x \in[0,1)$,

$$
\begin{aligned}
\lim _{n \rightarrow \infty} \frac{\sum_{k=1}^{n} \varepsilon_{k}(x, \beta)}{n} \\
\quad=\int_{0}^{1} \chi_{[1 / \beta, 1)}(x) d \mu_{\beta}(x)=\mu_{\beta}\left(\left[\frac{1}{\beta}, 1\right)\right)>0 .
\end{aligned}
$$

Note that $\mu_{\beta}$ is equivalent to the Lebesgue measure $\mathscr{L}$; then, for $\mathscr{L}$-a.e. $x$, the asymptotic density of $A_{\beta}(x)$

$$
\rho\left(A_{\beta}(x)\right)=\mu_{\beta}\left(\left[\frac{1}{\beta}, 1\right)\right)>0
$$

that is, $\mathscr{L}\left\{x \in[0,1): \rho\left(A_{\beta}(x)\right)=\mu_{\beta}([1 / \beta, 1))\right\}=1$. By Theorem 1 , we know

$$
\left\{x \in[0,1): \rho\left(A_{\beta}(x)\right)=\mu_{\beta}\left(\left[\frac{1}{\beta}, 1\right)\right)\right\} \subset \mathscr{F}_{\beta} .
$$

Thus $\mathscr{L}\left(\mathscr{F}_{\beta}\right)=1$.

Let $a, d \geq 1$ be natural numbers. Define

$$
\mathscr{I}_{\beta}(a, d):=\left\{x \in[0,1): \varepsilon_{a+k d}(x, \beta)=1 \forall k \geq 0\right\} .
$$

Then $\mathscr{I}_{\beta}=\cup_{(a, d)} \mathscr{I}_{\beta}(a, d)$, where $(a, d) \in \mathbb{N} \times \mathbb{N}$. To prove that $\mathscr{L}\left(\mathscr{I}_{\beta}\right)=0$, it suffices to show that $\mathscr{L}\left(\mathscr{I}_{\beta}(a, d)\right)=0$ for any $(a, d) \in \mathbb{N} \times \mathbb{N}$. In fact, let

$$
\mathscr{I}_{\beta}(a, d, m)=\left\{x \in[0,1): \varepsilon_{a+k d}(x, \beta)=1 \forall 0 \leq k \leq m\right\}
$$

and then

$$
\mathscr{I}_{\beta}(a, d)=\bigcap_{m=0}^{\infty} \mathscr{I}_{\beta}(a, d, m)
$$

The set $\mathscr{I}_{\beta}(a, d, 0)$ consists of finitely many cylinders $J\left(\varepsilon_{1}, \ldots, \varepsilon_{a-1}, \varepsilon_{a}\right)$ with $\varepsilon_{a}=1$. Now we estimate the length of $J\left(\varepsilon_{1}, \ldots, \varepsilon_{a-1}, \varepsilon_{a}\right)$ by the following two cases.

(i) If the word $\left(\varepsilon_{1}, \ldots, \varepsilon_{a-1}, 1\right)$ is admissible, then the cylinder $J\left(\varepsilon_{1}, \ldots, \varepsilon_{a-1}, 0\right)$ is full by Lemma 2.9(i) in [6] and consequently $\left|J\left(\varepsilon_{1}, \ldots, \varepsilon_{a-1}, 0\right)\right|=\beta^{-a}$. Since $\left|J\left(\varepsilon_{1}, \ldots, \varepsilon_{a-1}, 0\right)\right|+\left|J\left(\varepsilon_{1}, \ldots, \varepsilon_{a-1}, 1\right)\right|=\left|J\left(\varepsilon_{1}, \ldots, \varepsilon_{a-1}\right)\right|$, $\left|J\left(\varepsilon_{1}, \ldots, \varepsilon_{a-1}\right)\right| \leq \beta^{-(a-1)}$, and $1<\beta \leq 2$, we have

$$
\begin{aligned}
\frac{\left|J\left(\varepsilon_{1}, \ldots, \varepsilon_{a-1}, 1\right)\right|}{\left|J\left(\varepsilon_{1}, \ldots, \varepsilon_{a-1}\right)\right|} & =1-\frac{\beta^{-a}}{\left|J\left(\varepsilon_{1}, \ldots, \varepsilon_{a-1}\right)\right|} \\
& \leq 1-\frac{\beta^{-a}}{\beta^{-(a-1)}}=1-\frac{1}{\beta} \leq \frac{1}{2} .
\end{aligned}
$$

That is, $\left|J\left(\varepsilon_{1}, \ldots, \varepsilon_{a-1}, 1\right)\right| \leq(1 / 2)\left|J\left(\varepsilon_{1}, \ldots, \varepsilon_{a-1}\right)\right|$.

(ii) If the word $\left(\varepsilon_{1}, \ldots, \varepsilon_{a-1}, 1\right)$ is not admissible, then $J\left(\varepsilon_{1}, \ldots, \varepsilon_{a-1}, 0\right)=J\left(\varepsilon_{1}, \ldots, \varepsilon_{a-1}\right)$, which is not contained in $\mathscr{I}_{\beta}(a, d, 0)$.
Therefore,

$$
\begin{aligned}
\mathscr{L}\left(\mathscr{I}_{\beta}(a, d, 0)\right) & =\sum_{\left(\varepsilon_{1}, \ldots, \varepsilon_{a-1}, 1\right) \in \Sigma_{\beta}^{a}}\left|J\left(\varepsilon_{1}, \ldots, \varepsilon_{a-1}, 1\right)\right| \\
& \leq \frac{1}{2} \sum_{\left(\varepsilon_{1}, \ldots, \varepsilon_{a-1}\right) \in \Sigma_{\beta}^{a-1}}\left|J\left(\varepsilon_{1}, \ldots, \varepsilon_{a-1}\right)\right| \leq \frac{1}{2} .
\end{aligned}
$$

When $m=1$, note that $\mathscr{I}_{\beta}(a, d, 1) \subset \mathscr{I}_{\beta}(a, d, 0)$ and $\mathscr{I}_{\beta}(a, d, 1)$ consists of the cylinders $J\left(\varepsilon_{1}, \ldots\right.$, $\left.\varepsilon_{a-1}, 1, \varepsilon_{a+1}, \ldots, \varepsilon_{a+d-1}, 1\right) \quad$ with $\quad\left(\varepsilon_{1}, \ldots, \varepsilon_{a-1}, 1, \varepsilon_{a+1}, \ldots\right.$, $\left.\varepsilon_{a+d-1}, 1\right) \in \Sigma_{\beta}^{a+d}$. A similar argument with the estimate of $\mathscr{L}\left(\mathscr{I}_{\beta}(a, d, 0)\right)$ implies

$$
\begin{aligned}
& \sum_{\left(\varepsilon_{1}, \ldots, \varepsilon_{a-1}, 1, \varepsilon_{a+1}, \ldots, \varepsilon_{a+d-1}, 1\right) \in \Sigma_{\beta}^{a+d}} J\left(\varepsilon_{1}, \ldots, \varepsilon_{a-1}, 1, \varepsilon_{a+1}, \ldots, \varepsilon_{a+d-1}, 1\right) \\
& \leq \frac{1}{2}\left|J\left(\varepsilon_{1}, \ldots, \varepsilon_{a-1}, 1\right)\right|
\end{aligned}
$$

for any $\left(\varepsilon_{1}, \ldots, \varepsilon_{a-1}, 1\right) \in \Sigma_{\beta}^{a}$. Therefore,

$$
\mathscr{L}\left(\mathscr{I}_{\beta}(a, d, 1)\right) \leq \frac{1}{2} \mathscr{L}\left(\mathscr{I}_{\beta}(a, d, 0)\right) \leq \frac{1}{2^{2}} .
$$

Repeating this argument, we obtain

$$
\mathscr{L}\left(\mathscr{I}_{\beta}(a, d, m)\right) \leq \frac{1}{2^{m+1}},
$$

which implies $\mathscr{L}\left(\mathscr{I}_{\beta}(a, d)\right)=0$ together with (25). Then we complete the proof.

The following is a consequence of Theorem 11.

Corollary 12. Consider $\mathscr{L}\left(\mathscr{F}_{\beta} \backslash \mathscr{I}_{\beta}\right)=1$.

Lemma 13. Let $1<\beta \leq 2$ such that $S_{\beta}$ is a SFT. Then $\operatorname{dim}_{H} \mathscr{I}_{\beta}=1$.

Proof. Since $S_{\beta}$ is a SFT, there exists $N \in \mathbb{N}$ such that it is enough to forbid finitely many words of length $N-1$. Note that $\mathscr{I}_{\beta} \supset \mathscr{I}_{\beta}(1, d)$ for any $d \in \mathbb{N}$. Now we construct a Cantor subset $F$ of $\mathscr{I}_{\beta}(1, d)$ with large Hausdorff dimension when $d$ is large.

Let $d^{\prime}=d+1$ with $d>2 N$ and define

$$
\begin{gathered}
F=\left\{x \in[0,1): \varepsilon_{1+k d^{\prime}}=1,\right. \\
\varepsilon_{2+k d^{\prime}} \ldots \varepsilon_{N+1+k d^{\prime}}=\varepsilon_{2+d-N+k d^{\prime}} \ldots \varepsilon_{1+d+k d^{\prime}} \\
\left.=0^{N} \forall k \geq 0\right\},
\end{gathered}
$$

where $\varepsilon_{i}$ means $\varepsilon_{i}(x, \beta)$ for simplicity. Then the set $F$ can be regarded as a Cantor-like set as the following: the interval $F_{0}$ of level 0 is $[0,1)$ and the set $F_{i+1}$ of level $i+1$ consists of the cylinders $J\left(1,0^{N}\right.$, $\left.\varepsilon_{N+2} \ldots \varepsilon_{d-N+1}, 0^{N}, \ldots, 1,0^{N}, \varepsilon_{N+2+i d^{\prime}} \ldots \varepsilon_{d-N+1+i d^{\prime}}, 0^{N}\right)$ for 
any $i \geq 0$, where $\varepsilon_{N+2+j d^{\prime}} \ldots \varepsilon_{d-N+1+j d^{\prime}}$ is any admissible word for $0 \leq j \leq i$. Arrange such cylinders from left to right and denote them by $\mathscr{J}_{i, \ell}, 1 \leq \ell \leq i M$, where $M$ is the number of admissible words of length $d-2 N$; that is, $M=\# \Sigma_{\beta}^{d-2 N}$. Then

$$
F_{i}=\bigcup_{\ell} \mathscr{F}_{i, \ell}, \quad F=\bigcap_{i=0}^{\infty} F_{i} .
$$

Let $\mathscr{J}_{i, \ell}$ be any cylinder of level $i$ and let $\mathscr{J}_{i-1, \ell^{\prime}}$ be its father cylinder of level $i-1$. Note that both $\mathscr{J}_{i, \ell}$ and $\mathscr{J}_{i-1, \ell^{\prime}}$ are full cylinders; we know that their lengths are $\beta^{-i(d+1)}$ and $\beta^{-(i-1)(d+1)}$, respectively. So the ratio between $\left|\mathscr{F}_{i, \ell}\right|$ and $\left|\mathscr{F}_{i-1, \ell^{\prime}}\right|$ is $\beta^{-(d+1)}$. Therefore $F$ is a self-similar set. Obviously $F$ satisfies strong separation condition (SSC). By the Hausdorff dimension formula for the self-similar set with SSC (see Falconer [9], Page 130), we know that $\operatorname{dim}_{H} F$ is the unique solution $s$ of the equation

$$
\sum_{\ell=1}^{M}\left(\beta^{-(d+1)}\right)^{s}=1
$$

That is, $\operatorname{dim}_{H} F=(\log M) /(d+1) \log \beta$. Note that $M \geq \beta^{d-2 N}$; then

$$
\operatorname{dim}_{H} \mathscr{I}_{\beta} \geq \operatorname{dim}_{H} \mathscr{I}(1, d) \geq \operatorname{dim}_{H} F \geq \frac{d-2 N}{d+1} .
$$

We obtain $\operatorname{dim}_{H} \mathscr{I}_{\beta}=1$ by letting $d \rightarrow \infty$.

\section{Theorem 14. Consider}

(i) $\operatorname{dim}_{H} \mathscr{F}_{\beta}^{c}=0$;

(ii) $\operatorname{dim}_{H} \mathscr{I}_{\beta}=1$.

Proof. (i) Since $\mathscr{F}_{\beta}^{c} \subset\left\{x \in[0,1): \lim _{n \rightarrow \infty}\left(N_{n}(x, \beta) / n\right)=\right.$ $0\}=: E$, it suffices to show that the Hausdorff dimension of the right-hand side set is zero. In fact, note that $N_{n}(x, \beta)=$ $\sum_{k=0}^{n-1} \chi_{[1 / \beta, 1)}\left(T_{\beta}^{k} x\right)$; by the main theorem in [10], we have

$$
\begin{array}{r}
\operatorname{dim}_{H} E=\sup \left\{\frac{h_{\mu}\left(T_{\beta}\right)}{\log \beta}: \mu \text { is } T_{\beta}\right. \text {-invariant, } \\
\left.\mu\left(\left[\frac{1}{\beta}, 1\right)\right)=0\right\},
\end{array}
$$

where $h_{\mu}\left(T_{\beta}\right)$ is the measure-theoretical entropy of $T_{\beta}$ with respect to $\mu$. Since the $T_{\beta}$-invariant measure $\mu$ with $\mu([1 / \beta, 1))=0$ is just the Dirac measure at the origin 0 , the measure-theoretical entropy $h_{\mu}\left(T_{\beta}\right)=0$, which implies $\operatorname{dim}_{H} E=0$. Therefore $\operatorname{dim}_{H} \mathscr{F}_{\beta}^{c}=0$.

(ii) Let $1<\beta_{n}<\beta$ be a sequence of numbers satisfying $S_{\beta_{n}}$ which are SFTs and $\beta_{n} \rightarrow \beta$ when $n \rightarrow \infty$. Note that $\Sigma_{\beta_{n}} \subset$ $\Sigma_{\beta}$; write $H_{\beta}^{\beta_{n}}=\pi_{\beta}\left(\Sigma_{\beta_{n}}\right)$. Define the function $h: H_{\beta}^{\beta_{n}} \rightarrow$ $[0,1)$ as $h(x)=\pi_{\beta_{n}}(\varepsilon(x, \beta))$ for any $x \in H_{\beta}^{\beta_{n}}$.

We claim that $h\left(\mathscr{I}_{\beta}(1, d) \cap H_{\beta}^{\beta_{n}}\right)=\mathscr{I}_{\beta_{n}}(1, d)$. Indeed, on the one hand, for any $x \in \mathscr{I}_{\beta}(1, d) \cap H_{\beta}^{\beta_{n}}$, we know $\varepsilon\left(h(x), \beta_{n}\right)=\varepsilon(x, \beta)$ by the definition of the function $h$. Note that $x \in \mathscr{I}_{\beta}(1, d)$; then $h(x) \in \mathscr{I}_{\beta_{n}}(1, d)$, which leads to $h\left(\mathscr{I}_{\beta}(1, d) \cap H_{\beta}^{\beta_{n}}\right) \subset \mathscr{I}_{\beta_{n}}(1, d)$. On the other hand, for any $y \in \mathscr{I}_{\beta_{n}}(1, d)$, take $x=\pi_{\beta}\left(\varepsilon\left(y, \beta_{n}\right)\right) \in H_{\beta}^{\beta_{n}}$. Clearly $h(x)=y$ and $\varepsilon(x, \beta)=\varepsilon\left(y, \beta_{n}\right)$, which implies $x \in \mathscr{I}_{\beta}(1, d)$. So $\mathscr{I}_{\beta_{n}}(1, d) \subset h\left(\mathscr{I}_{\beta}(1, d) \cap H_{\beta}^{\beta_{n}}\right)$.

By Theorem 3.1(4) in [11], the function $h$ is $\left(\log \beta_{n} / \log \beta\right)$ Hölder which implies

$$
\begin{aligned}
\frac{\log \beta_{n}}{\log \beta} \operatorname{dim}_{H} \mathscr{I}_{\beta_{n}}(1, d) & =\frac{\log \beta_{n}}{\log \beta} \operatorname{dim}_{H} h\left(\mathscr{I}_{\beta}(1, d) \cap H_{\beta}^{\beta_{n}}\right) \\
& \leq \operatorname{dim}_{H}\left(\mathscr{I}_{\beta}(1, d) \cap H_{\beta}^{\beta_{n}}\right) .
\end{aligned}
$$

Since $\mathscr{I}_{\beta}(1, d) \cap H_{\beta}^{\beta_{n}} \subset \mathscr{I}_{\beta}(1, d)$, we have

$$
\operatorname{dim}_{H} \mathscr{I}_{\beta}(1, d) \geq \frac{\log \beta_{n}}{\log \beta} \operatorname{dim}_{H} \mathscr{I}_{\beta_{n}}(1, d),
$$

which concludes the result by letting $d \rightarrow \infty$ and then $n \rightarrow$ $\infty$, together with (34) in Lemma 13.

\section{Conflict of Interests}

The authors declare that there is no conflict of interests regarding the publication of this paper.

\section{Acknowledgments}

The authors thank the three anonymous reviewers for many helpful comments and suggestions that led to significant improvement for the paper. In addition, Bing Li was supported by NSFC (nos. 11201155 and 11371148) and Fundamental Research Funds for the Central Universities SCUT (2013ZZ0085). Chao Ma was supported by the Science and Technology Development Fund of Macau (no. 069/2011/A).

\section{References}

[1] E. Szemerédi, "On sets of integers containing no $k$ elements in arithmetic progression," in Proceedings of the International Congress of Mathematicians (ICM '74), vol. 2, pp. 503-504, Vancouver, Canada, 1974, Acta Arithmetica, vol. 27, pp. 199-245, 1975.

[2] B. Green and T. Tao, "The primes contain arbitrarily long arithmetic progressions," Annals of Mathematics, vol. 167, no. 2, pp. 481-547, 2008.

[3] S. S. Wagstaff Jr., "On sequence, of positive integers not containing arithmetical progressions," Proceedings of the American Mathematical Society, vol. 36, pp. 395-397, 1972.

[4] T. Šalát and J. Tomanová, "On sequences of positive integers containing arithmetical progressions," Mathematical Communications, vol. 10, no. 1, pp. 47-53, 2005.

[5] W. Parry, "On the $\beta$-expansions of real numbers," Acta Mathematica Academiae Scientiarum Hungaricae, vol. 11, pp. 401-416, 1960. 
[6] B. Li and J. Wu, "Beta-expansion and continued fraction expansion," Journal of Mathematical Analysis and Applications, vol. 339, no. 2, pp. 1322-1331, 2008.

[7] A. Rényi, "Representations for real numbers and their ergodic properties," Acta Mathematica Academiae Scientiarum Hungaricae, vol. 8, pp. 477-493, 1957.

[8] A. O. Gel'fond, "A common property of number systems," Izvestiya Akademii Nauk SSSR. Seriya Matematicheskaya, vol. 23, pp. 809-814, 1959 (Russian).

[9] K. J. Falconer, Fractal Geometry: Mathematical Foundations and Applications, John Wiley \& Sons, Chichester, UK, 2003.

[10] C.-E. Pfister and W. G. Sullivan, "Large deviations estimates for dynamical systems without the specification property. Applications to the $\beta$-shifts," Nonlinearity, vol. 18, no. 1, pp. 237-261, 2005.

[11] J.-C. Ban and B. Li, "The multifractal spectra for the recurrence rate of beta-transformations," Journal of Mathematical Analysis and Applications, vol. 420, no. 2, pp. 1662-1679, 2014. 


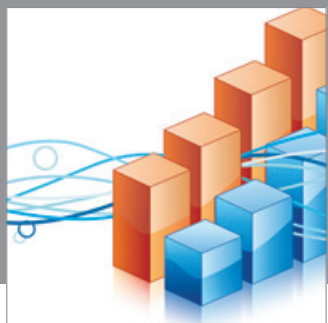

Advances in

Operations Research

mansans

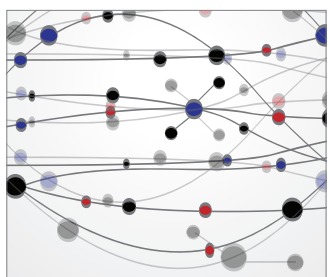

The Scientific World Journal
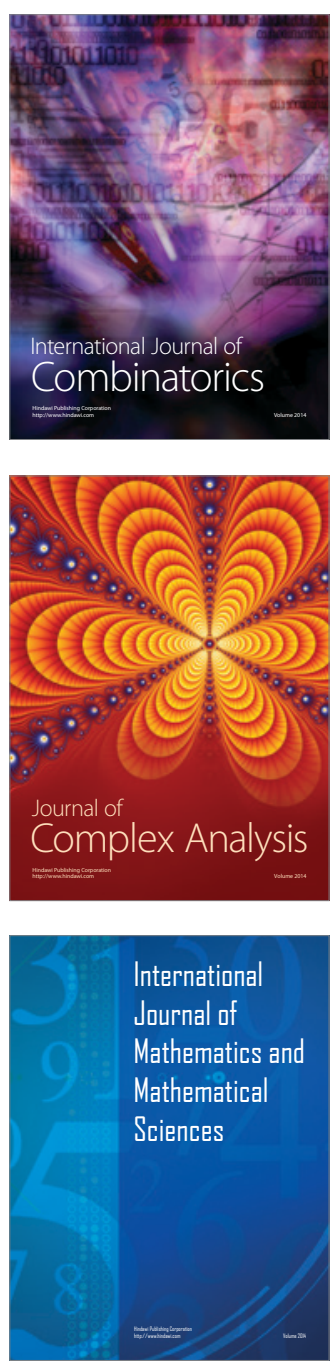
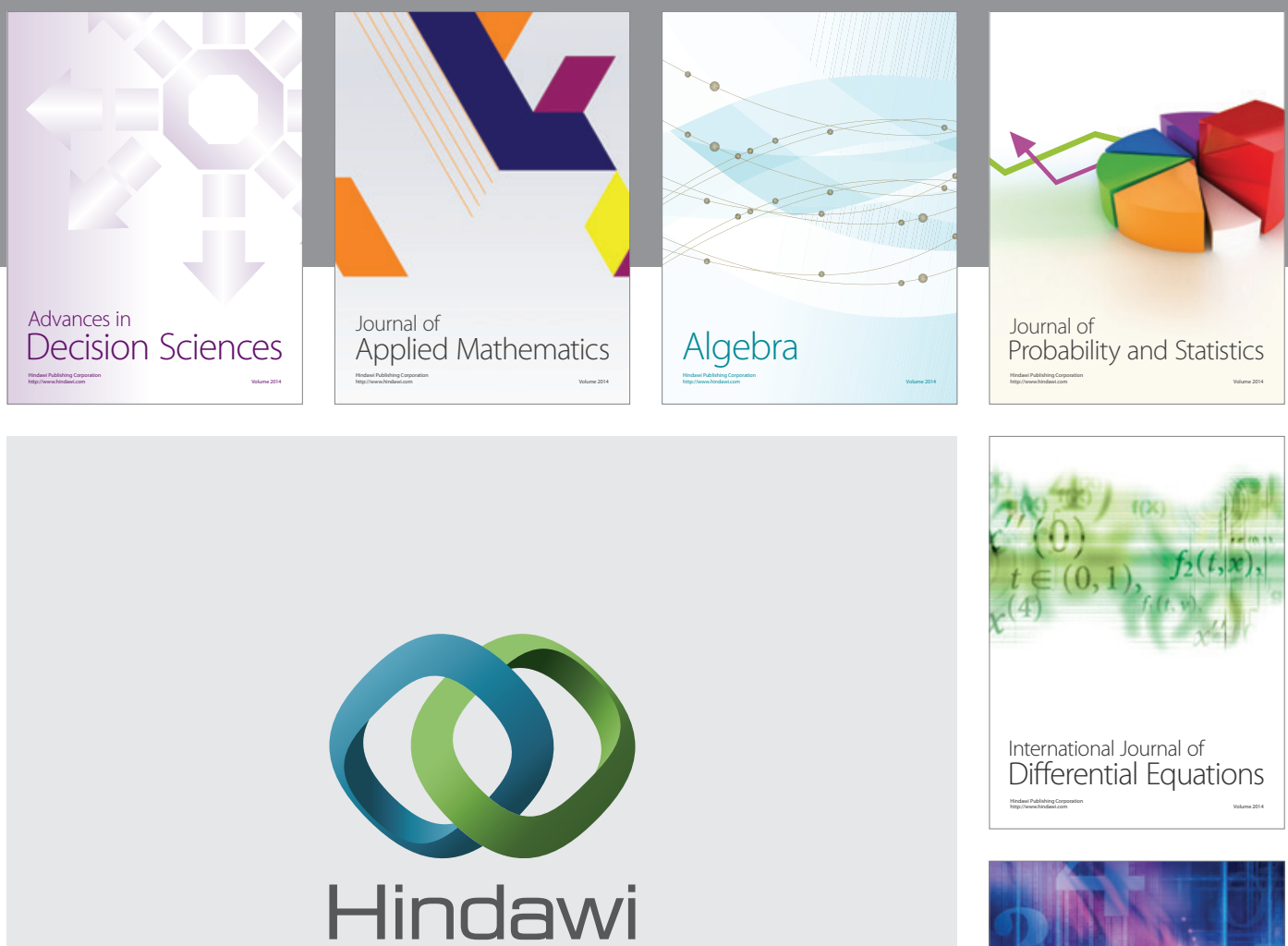

Submit your manuscripts at http://www.hindawi.com
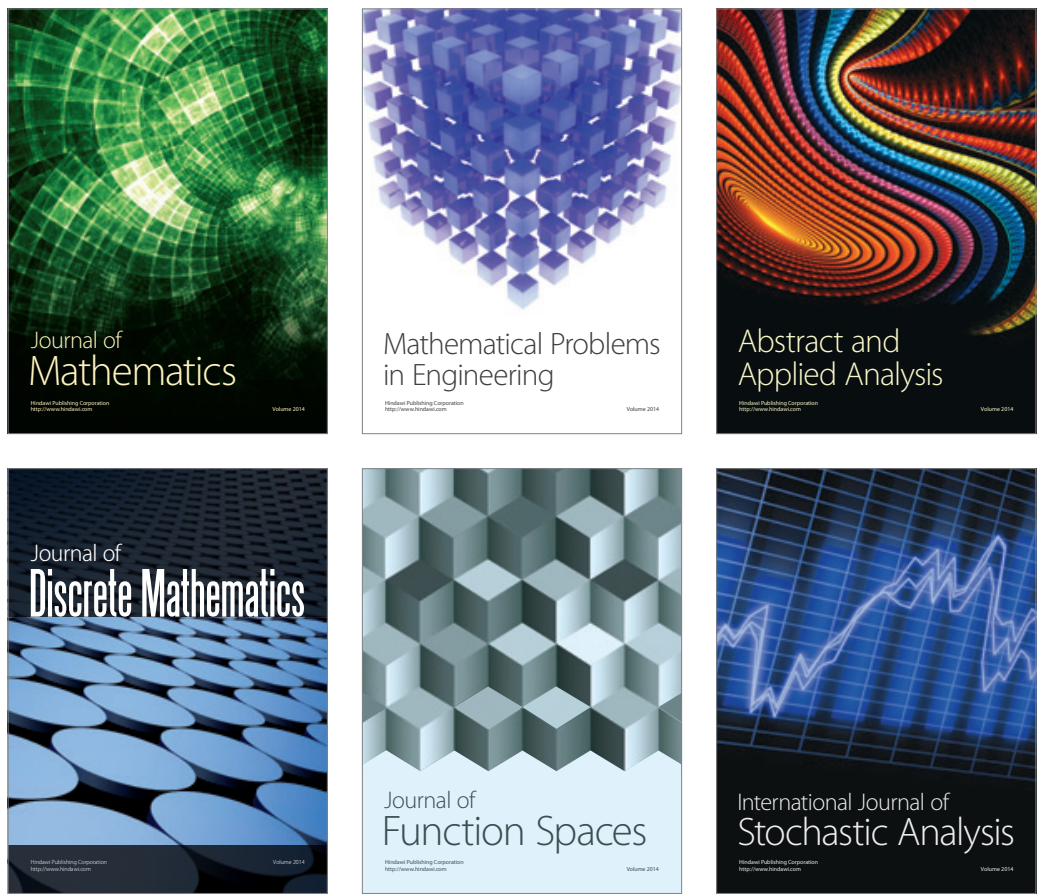

Journal of

Function Spaces

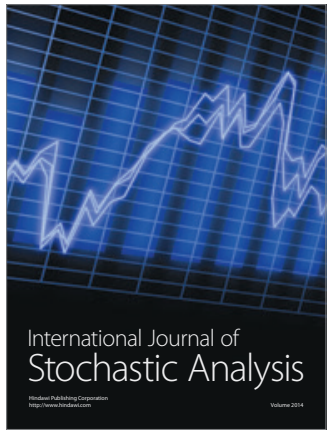

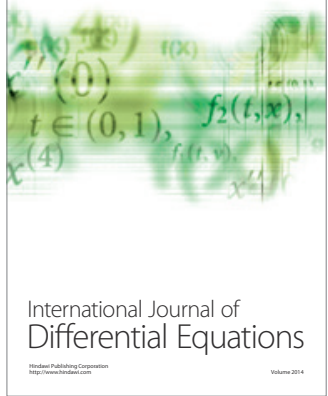
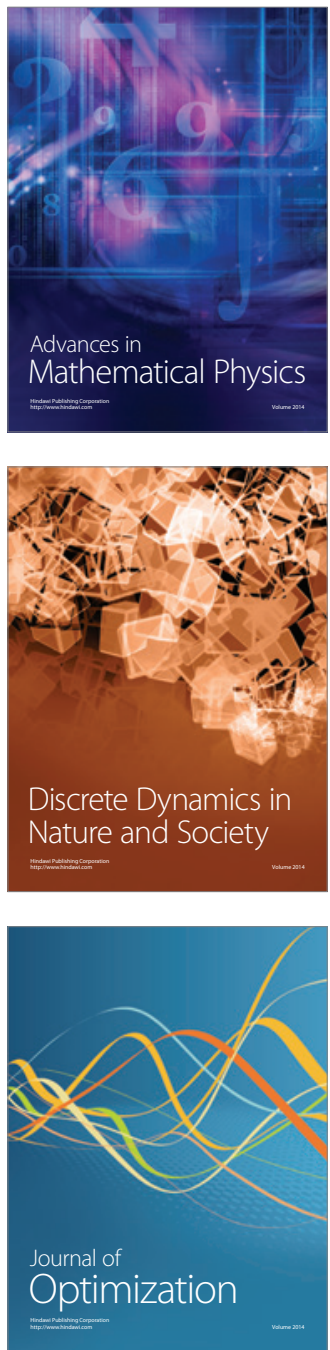